\title{
Comparison of time-resolved and continuous-wave near-infrared techniques for measuring cerebral blood flow in piglets
}

\author{
Mamadou Diop \\ Kenneth M. Tichauer \\ Jonathan T. Elliott \\ Lawson Health Research Institute \\ Imaging Program \\ London, Ontario, Canada N6A 4V2 \\ and \\ University of Western Ontario \\ Department of Medical Biophysics \\ London, Ontario, Canada N6G 2V4
}

\section{Mark Migueis}

Lawson Health Research Institute

Imaging Program

London, Ontario, Canada N6A 4V2

\author{
Ting-Yim Lee \\ Lawson Health Research Institute \\ Imaging Program \\ London, Ontario, Canada N6A 4V2 \\ and \\ University of Western Ontario \\ Department of Medical Biophysics \\ London, Ontario, Canada N6G 2V4 \\ and \\ Robarts Research Institute \\ Imaging Research Laboratories \\ London, Ontario, Canada N6A 5K8
}

\begin{abstract}
A primary focus of neurointensive care is monitoring the injured brain to detect harmful events that can impair cerebral blood flow (CBF), resulting in further injury. Since current noninvasive methods used in the clinic can only assess blood flow indirectly, the goal of this research is to develop an optical technique for measuring absolute CBF. A time-resolved near-infrared (TR-NIR) apparatus is built and CBF is determined by a bolus-tracking method using indocyanine green as an intravascular flow tracer. As a first step in the validation of this technique, CBF is measured in newborn piglets to avoid signal contamination from extracerebral tissue. Measurements are acquired under three conditions: normocapnia, hypercapnia, and following carotid occlusion. For comparison, CBF is concurrently measured by a previously developed continuous-wave NIR method. A strong correlation between CBF measurements from the two techniques is revealed with a slope of $0.79 \pm 0.06$, an intercept of $-2.2 \pm 2.5 \mathrm{ml} / 100 \mathrm{~g} / \mathrm{min}$, and an $\mathrm{R}^{2}$ of $0.810 \pm 0.088$. Results demonstrate that TR-NIR can measure CBF with reasonable accuracy and is sensitive to flow changes. The discrepancy between the two methods at higher CBF could be caused by differences in depth sensitivities between continuous-wave and time-resolved measurements. ๑ 2010 Society of Photo-Optical Instrumentation Engineers. [DOI: 10.1117/1.3488626]
\end{abstract}

Keywords: cerebral blood flow; cerebral hemodynamics; time-resolved reflectance; near-infrared spectroscopy; indocyanine green.

Paper 10187R received Apr. 9, 2010; revised manuscript received Jul. 23, 2010; accepted for publication Jul. 28, 2010; published online Sep. 16, 2010.

Keith St. Lawrence

Lawson Health Research Institute Imaging Program

London, Ontario, Canada N6A 4V2 and

University of Western Ontario

Department of Medical Biophysics

London, Ontario, Canada N6G 2V4

\section{Introduction}

The goal of neurointensive care is to provide an optimal environment for recovery from life-threatening neurological emergencies such as stroke, traumatic brain injury, and subarachnoid hemorrhage. ${ }^{1,2}$ Owing to the brain's high energy demands and limited substrate storage, one of the primary concerns is preventing secondary cerebral ischemia due to systemic (hypotension and hypocapnia) or intracranial (edema, hypertension, and impaired autoregulation) factors. This focus has led to the recognition that a monitoring device capable of detecting cerebral ischemia would greatly enhance the management of neurological emergencies.

Address all correspondence to: Mamadou Diop, Lawson Health Research Institute, Imaging Program, London, Ontario, Canada N6A 4V2. Tel: 519-646-6000 ext. 64166; Fax: 519-646-6110. E-mail: mdiop@lawsonimaging.ca
Techniques for measuring cerebral blood flow (CBF) using clinical imaging modalities, e.g., computed tomography (CT) and magnetic resonance imaging (MRI), have been developed, but the need to transfer critically ill patients to imaging facilities and prolonged scanning times seriously impede their use in the management of intensive care patients. ${ }^{3}$ The primary clinical method for bedside blood flow monitoring is transcranial Doppler (TCD), which measures mean flow velocities in cerebral arteries. However, the accuracy of TCD is highly operator dependent, the signal is not found in 10 to $30 \%$ of patients, and the technique cannot measure blood flow in the microvasculature, which can be different from flow in major arteries. ${ }^{4}$ Cerebral blood flow can be measured directly using laser Doppler or thermal diffusion flowmetry, ${ }^{5,6}$ but these techniques are invasive, requiring insertion of probes

1083-3668/2010/15(5)/057004/10/\$25.00 @ 2010 SPIE 
directly into tissue, and they can only measure CBF in the small volume where the probes are placed. Wider applicability requires noninvasive techniques that can assess $\mathrm{CBF}$ in multiple regions.

Optical methods are promising alternatives to current invasive techniques, since they are safe, portable, and provide measurements of tissue oxygenation and hemodynamics. ${ }^{7,8}$ Our group has developed a continuous-wave $(\mathrm{CW})$, nearinfrared spectroscopy (NIRS) technique for measuring CBF in newborns. ${ }^{9-11}$ The technique is based on bolus-tracking theory $^{12}$ and uses the near-infrared light absorbing dye indocyanine green (ICG) as an intravascular contrast agent. The major challenge in adapting our ICG bolus-tracking technique to adult patients is the sizable signal contamination from superficial tissue. It has been previously reported that the ICG bolus-tracking method combined with CW-NIRS underestimates $\mathrm{CBF}$ in adults by a factor of 3 due primarily to the overestimation of the optical path length through brain tissue. $^{13,14}$

Improved depth sensitivity has been achieved using photon time-of-flight information measured directly by time-resolved (TR) techniques, or indirectly through phase changes measured by frequency-domain (FD) techniques. ${ }^{15-18}$ The depth sensitivity made available by these techniques is due to the fact that early arriving photons travel shorter distances (i.e., through superficial tissue), while late arriving photons travel longer distances (i.e., deeper into brain tissue). Both approaches have been used to extract first-pass kinetics of ICG through the brain in adults. ${ }^{19,20}$ Although promising, these studies were qualitative and did not attempt to determine absolute CBF. The ultimate goal of our research is to measure $\mathrm{CBF}$ in critical-care patients by adapting our quantitative ICG bolus-tracking method to TR reflectance measurements. A time-correlated single-photon counting system was assembled and, as a first step, we investigated the ability of the system to measure $\mathrm{CBF}$ over a range of values in piglets. Piglets were chosen to avoid signal contamination from extracerebral tissue, which enabled TR reflectance data to be modeled by the solution to the diffusion equation for a homogeneous semiinfinite geometry. ${ }^{21}$ Absolute concentration of ICG in brainrequired to measure $\mathrm{CBF}$ - was determined from the change in the absorption coefficient. For comparison, CBF was concurrently measured using our previously developed CW system.

\section{Materials and Methods}

\subsection{Instrumentation}

The light source of the TR system consisted of a picosecond pulsed diode laser (LDH-P-C-810, PicoQuant, Germany) emitting at $802 \mathrm{~nm}$, which coincides with the peak absorption wavelength of ICG in plasma, and a computer-controlled laser driver (PDL 828, PicoQuant). The laser output power and pulse repetition rate were set to $1.4 \mathrm{~mW}$ and $80 \mathrm{MHz}$, respectively. The laser output was coupled by a microscope objective lens $(\mathrm{NA}=0.25$, magnification $=10 \times)$ into a $1.5-\mathrm{m}$-long multimode fiber probe $(\mathrm{NA}=0.22$, core $400 \mu \mathrm{m}, 4.7-\mathrm{mm}$ outer diameter; Fiberoptics Technology, Pomfret, Connecticut) mounted on a custom-made holder. The average power delivered to a sample was attenuated to roughly $20 \mu \mathrm{W}$ using two variable neutral density filters (NDC-50-4M, Thorlabs, Newton, New Jersey).

Diffusely reflected light from the sample was collected by a 2-m fiber optic bundle (NA $=0.55,3.6-\mathrm{mm}$-diameter active area, and 4.7-mm outer diameter, Fiberoptics Technology) whose distal end was secured in front of an electromechanical shutter (SM05, Thorlabs). Light transmitted through the shutter was collected by a Peltier-cooled photomultiplier tube (PMT) (PMC-100, Becker and Hickl, Germany). Detection of single photons generated electrical pulses (amplitude of 50 to $200 \mathrm{mV}$, width $=1.5 \mathrm{~ns}$ ) that were transmitted to a time-correlated single photon counting (TCSPC) module (SPC-134, Becker and Hickl, Germany). The TCSPC module had a dead-time of $100 \mathrm{~ns}$, and because of that, the maximum count rate was constrained to approximately $800 \mathrm{kHz}$ (i.e., one electron detected every $1.25 \mu \mathrm{s}$ ) to minimize dead-time effects. This count rate, which was $1 \%$ of the laser pulse repetition rate, also minimized pile-up effects. ${ }^{22}$

To accurately quantify optical properties, the instrument response function (IRF) was measured to account for dispersion by the TR system. ${ }^{23}$ The IRF was measured by placing a thin piece of white paper between the emission fiber and the detection fiber bundle. The paper was coated with black toner to reduce back-reflection from the emission fiber. ${ }^{24,25}$ These measurements were acquired at the start of each animal experiment (described in Sec. 2.7) and at the same count rate $(800 \mathrm{kHz})$.

The CW broadband near-infrared apparatus has been described in detail elsewhere. ${ }^{9,10}$ Briefly, the illumination unit was a 50-W halogen light bulb, bandpass filtered from 600 to $1000 \mathrm{~nm}$. The emission and detection fiber optic bundles had a length of $2 \mathrm{~m}$, an NA of 0.55 , a 3.6-mm-diameter active area, and 4.7- $\mathrm{mm}$ outer diameter. The spectrometer consisted of a holographic grating housed in a light-tight box, which was coupled to a cooled chargecoupled device (CCD) camera (Wright Instruments, Middlesex, United Kingdom).

\subsection{Bolus-Tracking Method}

The hemodynamics of an organ can be characterized by tracking the passage of a bolus of contrast agent, in this case ICG, through the vascular bed. ${ }^{12}$ As such, the time-dependent concentration of ICG in tissue $Q(t)$ is related to the ICG concentration in arterial blood $C_{a}(t)$ by the following expression:

$$
Q(t)=\mathrm{CBF} \cdot \int_{0}^{t} C_{a}(u) R(t-u) d u .
$$

In Eq. (1), $R(t)$ is referred to as the impulse residue function, which represents the tissue ICG concentration for the idealized case that the input function $\mathrm{CBF} \cdot C_{a}(t)$ is defined by a dirac-delta function with unit mass deposited at time zero. A necessary assumption is that blood flow remains constant during the bolus-tracking experiment, which typically lasts about $40 \mathrm{~s}$. In the general case, ICG is injected in a peripheral vein, $Q(t)$ is measured by a NIR technique, and $C_{a}(t)$ is measured noninvasively by a dye densitometer (DDG 2001, Nihon Kohden, Japan). The function CBF $R(t)$ is extracted from the arterial and tissue ICG concentration curves using a deconvo- 
lution algorithm. ${ }^{9}$ The initial height of the derived function is $\mathrm{CBF}$, and the area under the curve is the cerebral blood volume (CBV). ${ }^{12}$

\subsection{Continuous-Wave Measurement of $Q(t)$}

For the CW-NIRS experiments, the modified Beer-Lambert law was used to determine $Q(t)$ from the change in the light attenuation spectrum $\Delta A(\lambda, t)$ :

$$
Q(t)=\Delta A(\lambda, t) /\left[\varepsilon_{\mathrm{ICG}}(\lambda) \cdot D P\right] ;
$$

where $\varepsilon_{\mathrm{ICG}}(\lambda)$ is the extinction coefficient spectrum of ICG, and $D P$ is the differential path length. ${ }^{26}$ The latter was determined from the $840-\mathrm{nm}$ water feature, using secondderivative spectroscopy to remove the effects of light scatter, and assuming a water concentration in brain of $85 \% .^{27}$

\subsection{Time-Resolved Measurement of $Q(t)$}

Quantification of the optical properties (i.e., the absorption coefficient $\mu_{a}$ and the reduced scattering coefficient $\mu_{s}^{\prime}$ ) was obtained using the solution to the diffusion equation for a homogeneous semi-infinite medium. ${ }^{21}$ The model solution was first convolved with the measured IRF, and then a nonlinear optimization routine (MATLAB function fminsearch) was used to fit the convolved model to each measured temporal point spread function (TPSF). The fitting range was set to $80 \%$ of the peak value on the leading edge and $20 \%$ on the falling edge. ${ }^{28}$ Determining the tissue ICG concentration first required measuring the baseline optical properties $\left(\mu_{a}\right.$ and $\left.\mu_{s}^{\prime}\right)$ from the TPSFs collected over a 10-s period prior to an ICG injection. The fitting procedure included an additional scaling factor to account for the laser power, detection gain, and coupling efficiency. ${ }^{23}$ The change in light absorption caused by ICG was then characterized using only $\mu_{a}$ as a fitting parameter, with $\mu_{s}^{\prime}$ and the scaling factor fixed to their baseline values. Finally, the measured change in the absorption coefficient $\left(\mu_{a}\right)$ was used to calculate ICG concentration by:

$$
Q(t)=\left[\mu_{a}(t)-\mu_{a}(0)\right] /\left[\ln (10) \times \varepsilon_{\mathrm{ICG}}\right],
$$

where $\mu_{a}(0)$ represents the baseline value determined prior to the injection of ICG, and $\varepsilon_{\mathrm{ICG}}$ is the extinction coefficient of ICG at $802 \mathrm{~nm}\left(186 \mathrm{OD} / \mathrm{mM} / \mathrm{cm}^{29}\right)$.

For comparison with the second-derivative, water-based measurements of DP, the differential path length was calculated from the difference between the mean transit times of the experimental TPSF and the IRF. ${ }^{30}$ A refractive index of 1.4 was used to convert the mean transit time measurements to $\mathrm{DP}^{31}$

\subsection{Time-Resolved Error Analysis}

A number of potential sources of error can reduce the accuracy of TR measurements, including improper characterization of instrument dispersion, timing uncertainties, and potential nonlinearities of the TCSPC system. ${ }^{23}$ The importance of these factors was assessed by determining how an error in each altered the expected change in $\mu_{a}$ corresponding to the difference between peak and baseline tissue ICG concentrations, denoted $\Delta \mu_{a}^{\max }$. We focused on $\Delta \mu_{a}^{\max }$, since the tissue concentration of ICG is directly proportional to $\mathrm{CBF}$
[Eq. (1)]. Two simulated TPSFs were generated from the homogeneous analytical model using typical baseline $\mu_{a}$ and $\mu_{s}^{\prime}$ values $\left(0.150 \mathrm{~cm}^{-1}\right.$ and $\left.7.4 \mathrm{~cm}^{-1}\right)$ and $\Delta \mu_{a}^{\max }=0.045 \mathrm{~cm}^{-1}$. The TPSFs were convolved with an IRF with a systematic error. These data were reanalyzed using the true IRF to determine the best-fit estimate of $\Delta \mu_{a}^{\max }$, which was then compared to the known value.

First, errors caused by instrument dispersion were assessed by changing the full width at half maximum (FWHM) of the measured IRF $( \pm 20 \%)$. Second, errors caused by timing uncertainties were assessed by shifting the position of the IRF by $\pm 21 \mathrm{ps}$, which was approximately five times larger than the uncertainty in position $(4.3 \mathrm{ps})$. $^{32}$ Finally, potential distortions in the shape of the TPSF caused by nonlinearities were characterized using two IRFs: one acquired at the baseline count rate of $800 \mathrm{kHz}$ and another acquired at $400 \mathrm{kHz}$, which corresponds to the maximum decrease following an injection of ICG. The TPSF representing baseline ICG concentration was convolved with the IRF measured at $800 \mathrm{kHz}$, and the TPSF corresponding to the peak tissue ICG concentration was convolved with the IRF measured at $400 \mathrm{kHz}$.

\subsection{Time-Resolved Phantom Experiments}

To test the accuracy of the TR system, the optical properties of a tissue-mimicking phantom (ISS, Champaign, Illinois) were measured. Thirty-two TPSFs with an integration time of $400 \mathrm{~ms}$ were acquired. The known $\mu_{a}$ and $\mu_{s}^{\prime}$ values provided by the manufacturer were 0.103 and $10.6 \mathrm{~cm}^{-1}$ at $690 \mathrm{~nm}$, and 0.101 and $9.6 \mathrm{~cm}^{-1}$ at $830 \mathrm{~nm}$, respectively.

\subsection{Animal Experiments}

All animal experiments were approved by the Animal Use Subcommittee of the Canadian Council on Animal Care at the University of Western Ontario. Duroc-cross piglets were delivered from a local supplier on the morning of the experiment. Piglets were anesthetized with $1.75 \%$ isoflurane (3\% during surgery), tracheotomized, and mechanically ventilated on an oxygen/medical air mixture. An ear vein was catheterized for ICG (Sigma-Aldrich, Saint Louis, Missouri) injections, and a femoral artery was catheterized to monitor blood pressure and intermittently collect blood samples for gas and glucose analysis. Two incisions were made lateral to the trachea, and vascular occluders (1.5 mm inner diameter, In Vivo Metric, Healdberg, California) were placed around both carotid arteries proximal to the carotid bifurcation. The occluders were used to temporarily create conditions of cerebral ischemia. ${ }^{33}$ Animals were placed in a prone position, and a custom-made probe holder was strapped to the head to hold the emission and detection probes $2.7 \mathrm{~cm}$ apart, parasagittally, approximately $1.5 \mathrm{~cm}$ dorsal to the eyes. Piglets were allowed to stabilize for $1 \mathrm{~h}$ after surgery before any CBF measurements were acquired. This delay was also sufficient to minimize drift artifacts in the TR measurements due to temperature changes. ${ }^{32}$

Cerebral blood flow was measured with both the TR and $\mathrm{CW}$ techniques, sequentially (separated by 5 to $10 \mathrm{~min}$ ) during normocapnia, hypercapnia, and temporary carotid occlusion. This required swapping the probes, which had the same outer diameter, from the two systems between measurements. For each animal, three sets of TR and CW data were acquired 
Table 1 Physiological parameters during normocapnia, hypercapnia, and carotid occlusion. Values are mean \pm SE. ${ }^{*} p<0.05$ compared with normocapnia.

\begin{tabular}{lccc}
\hline & Normocapnia & Hypercapnia & $\begin{array}{c}\text { Carotid } \\
\text { occlusion }\end{array}$ \\
\hline Heart rate (beats/min) & $145 \pm 5$ & $159 \pm 5$ & $159 \pm 6$ \\
$\begin{array}{l}\text { Mean arterial pressure } \\
(\mathrm{mmHg})\end{array}$ & $40.5 \pm 1.5$ & $40.0 \pm 1.3$ & $39.0 \pm 1.3$ \\
Arterial $\mathrm{PaCO}_{2}(\mathrm{mmHg})$ & $38.2 \pm 1.2$ & $50.9 \pm 2.0 *$ & $45.2 \pm 3.4$ \\
Arterial $\mathrm{O}_{2}$ saturation $(\%)$ & $99.6 \pm 0.2$ & $99.4 \pm 0.2$ & $99.7 \pm 0.2$ \\
Temperature $\left({ }^{\circ} \mathrm{C}\right)$ & $38.4 \pm 0.1$ & $38.3 \pm 0.1$ & $38.3 \pm 0.1$ \\
\hline
\end{tabular}

during normocapnia, two sets during hypercapnia, and a final two sets after vascular occlusion. The arterial ICG concentration curve was measured by attaching the dye densitometer probe to a forefoot. The bolus of ICG was $0.05 \mathrm{mg}$ dissolved in $0.5-\mathrm{ml}$ sterile water. Tissue concentration curves were determined from reflectance data acquired continually for $70 \mathrm{~s}$ at a sampling interval of $400 \mathrm{~ms}$ for the TR system and $200 \mathrm{~ms}$ for the CW apparatus. For the TR measurements, the initial count rate was adjusted to $800 \mathrm{kHz}(1 \%$ of the laser repetition rate). Each experiment was completed within $4 \mathrm{~h}$.

\subsection{Statistical Analysis}

All data are presented as mean \pm SE unless otherwise stated. Statistical analyses were conducted using SPSS 16.0 (SPSS, Chicago, Illinois) and statistical significance was defined as $p<0.05$. One-way analysis of variance (ANOVA) with post hoc analysis by Tukey's honestly significant difference (HSD) tests was used to assess any changes in a physiological parameter between the three conditions (normocapnia, hypercapnia, and carotid occlusion). A two-way repeated-measures ANOVA was used to assess differences in each of the nearinfrared measurements (CBF, $\mathrm{CBV}$, and $\mathrm{DP})$ with condition as the within-subjects variable and NIR technique as the between-subjects variable.

A common regression analysis could not be used to determine the statistical significance of correlations between $\mathrm{CBF}$ measurements from the two optical techniques, because multiple measurements were acquired from each animal. Instead, a variation of the generalized estimating equation technique was utilized. ${ }^{34,35}$ First, a linear fit was applied to the data from each piglet individually. Second, a significant correlation was tested by using a t-test to compare the average of the distribution of slopes against the null hypothesis (i.e., slope $=0$ ). Finally, the distribution of slopes was compared to a slope of 1 to determine the agreement with the line of identity. BlandAltman analysis was used to assess the agreement between CBF measurements from the two techniques. ${ }^{36}$

\section{Results}

Cerebral blood flow measurements were acquired in six piglets less than $24 \mathrm{~h}$ of age (mean weight $=1.52 \pm 0.06 \mathrm{~kg}$, five females and one male). Average physiological parameters dur-

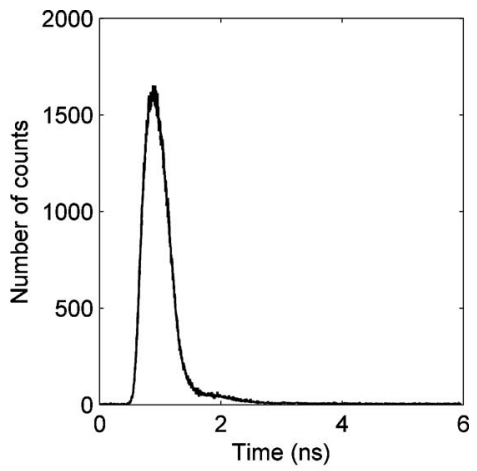

(a)

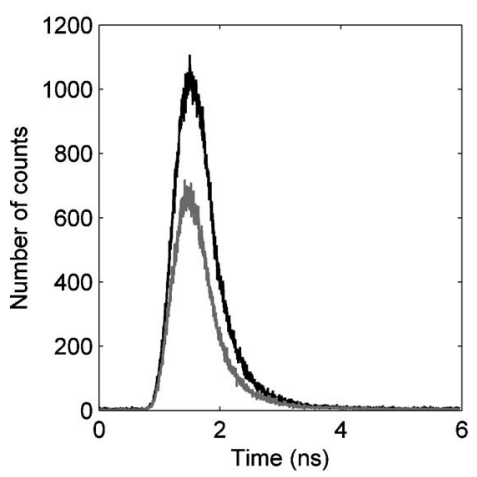

(b)

Fig. 1 (a) A typical instrument response function with a FWHM of $0.490 \mathrm{~ns}$. (b) Two experimental temporal point spread functions acquired over $400 \mathrm{~ms}$; these curves were acquired from the same piglet during one ICG run. The black curve was acquired prior to ICG injection, and the gray curve was acquired at the maximum reflectance change following the injection. Light absorption by ICG caused the profile of the TPSF to become narrower, as well as reduced the amplitude.

ing normocapnia, hypercapnia, and carotid occlusion are summarized in Table 1 . As expected, $\mathrm{PaCO}_{2}$ was significantly different between normocapnia and hypercapnia $(p<0.005)$. The mean $\mathrm{PaCO}_{2}$ value during carotid occlusion was similar to the mean hypercapnic value, since carotid occlusion was performed immediately following hypercapnia. No statistically significant differences were observed for any of the other physiological parameters. In total, 42 sets of TR- and CW-NIRS ICG measurements were completed. Two sets were removed because the arterial ICG concentration curves were not properly transferred from the DDG to the personal computer.

An example IRF is shown in Fig. 1(a). The FWHM characterizing the dispersion in the TR system was $0.490 \pm 0.001 \mathrm{~ns}$. The measured $\mu_{a}$ and $\mu_{s}^{\prime}$ values of the tissue-mimicking phantom were $0.118 \pm 0.001 \mathrm{~cm}^{-1}$ and $8.12 \pm 0.13 \mathrm{~cm}^{-1}$, respectively (mean $\pm \mathrm{SD}$ ). Two TPSFs measured from one piglet are shown in Fig. 1(b), each acquired over a 400-ms integration period. One was acquired at baseline, and the other corresponds to the largest reduction in the measured light reflectance following an injection of ICG. These data illustrate the typical change in amplitude and shape of the TPSF due to the passage of ICG through brain. The optical properties of the piglet head were determined 
Table 2 Mean differential path length (DP) measurements acquired by time-resolved (TR) and continuous-wave (CW) techniques during normocapnia, hypercapnia, and carotid occlusion. Values are mean \pm SE. ${ }^{*} p<0.05$ compared with TR values.

\begin{tabular}{ccc}
\hline & \multicolumn{2}{c}{ Mean DP $(\mathrm{cm})$} \\
\cline { 2 - 3 } Condition & $\mathrm{TR}$ & $\mathrm{CW}$ \\
\hline Baseline & $13.02 \pm 0.24$ & $9.98 \pm 0.59 *$ \\
Hypercapnia & $12.76 \pm 0.17$ & $9.96 \pm 0.45^{*}$ \\
Occulsion & $13.03 \pm 0.33$ & $10.10 \pm 0.45^{*}$ \\
\hline
\end{tabular}

from the baseline TPSF acquired prior to any ICG injection. Average values of $\mu_{a}$ and $\mu_{s}^{\prime}$ were $0.176 \pm 0.001 \mathrm{~cm}^{-1}$ and $7.47 \pm 0.04 \mathrm{~cm}^{-1}$, respectively.

The average DP values for the NIR techniques are provided in Table 2 for normocapnic, hypercapnic, and carotid occlusion conditions. A repeated-measures ANOVA determined that the DP values from the two techniques were significantly different across the three conditions $\left(\mathrm{F}_{1,10}=29.8\right.$, $p<0.001$, power $>0.99$ ), however, no significant interactions were observed between DP and condition with either technique, indicating that DP did not change significantly between conditions.

Figure 2 displays two sets of ICG concentration curves measured by the TR and CW-NIRS techniques. In this example, the peak tissue ICG concentration measured by CWNIRS was higher than that measured by the TR system. This difference translated into a higher CBF estimate determined by CW-NIRS $(82 \mathrm{ml} / 100 \mathrm{~g} / \mathrm{min})$ than by the TR method $(61 \mathrm{ml} / 100 \mathrm{~g} / \mathrm{min})$. The contrast-to-noise ratio $(\mathrm{CNR})$ for each NIR approach was calculated as the maximum signal change following ICG injection divided by the standard deviation of the background signal. The mean CNR was $116 \pm 26$ for the TR data, and $186 \pm 26$ for the CW-NIRS data (the latter was adjusted to account for the shorter integration time). These values were calculated from ICG concentration curves acquired during normocapnia, and the mean CNR was statistically greater for the CW data compared to the TR data $(p<0.01)$.

The correlation plot between CBF measurements from the two techniques and the corresponding Bland-Altman plot are shown in Fig. 3. The average slope, intercept, and $\mathrm{R}^{2}$ value from the individual regression analyses were $0.79 \pm 0.06$, $-2.2 \pm 2.5 \mathrm{ml} / 100 \mathrm{~g} / \mathrm{min}$, and $0.810 \pm 0.088$, respectively. The average intercept was not significantly different from zero. The average slope was significantly different from the null $(p<0.001)$, indicating that a correlation existed between the two sets of CBF measurements. However, the slope was also significantly different from unity $(p<0.05)$, indicating a difference between CBF measurements from the techniques as flow increased. This discrepancy was reflected in a significant main effect between techniques determined by the repeatedmeasures ANOVA $\left(\mathrm{F}_{1,10}=5.78, p<0.05\right.$, power $\left.>0.55\right)$ and in the mean $\mathrm{CBF}$ difference $(16.1 \mathrm{ml} / 100 \mathrm{~g} / \mathrm{min})$ revealed by the Bland-Altman analysis $(p<0.001)$.

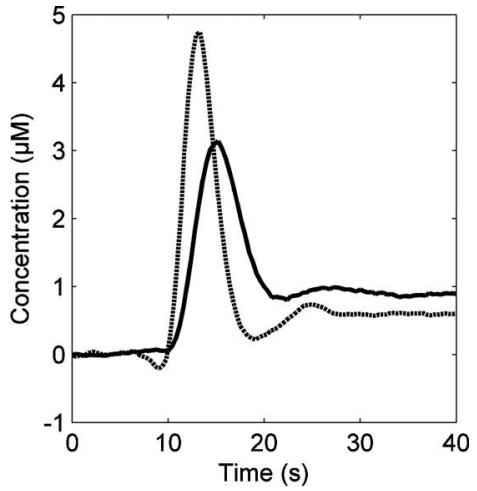

(a)

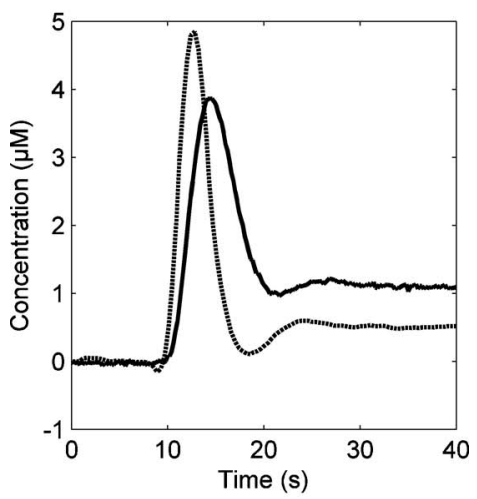

(b)

Fig. 2 Representative tissue (solid line) and arterial blood (dotted line) ICG concentration curves measured simultaneously following an intravenous bolus injection of ICG $(0.1 \mathrm{mg} / \mathrm{ml})$ using (a) the TR-NIR system and (b) the CW-NIRS apparatus. Both sets of curves were acquired sequentially from the same animal with a delay of approximately $10 \mathrm{~min}$ between ICG injections. For viewing purposes, the tissue concentration curves were scaled by a factor of 30 to compensate for the small blood volume in the brain.

The correlation plot between CBV measurements from the two techniques is shown in Fig. 4, along with the corresponding Bland-Altman plot. The average slope, intercept, and $\mathrm{R}^{2}$ from the individual regression analyses were $0.38 \pm 0.16$, $1.72 \pm 0.65 \mathrm{ml} / 100 \mathrm{~g}$, and $0.33 \pm 0.13$, respectively. Similar to the $\mathrm{CBF}$ results, the average slope was significantly greater than the null $(p<0.05)$ and less than unity $(p<0.025)$. A significant main effect between NIR techniques was found $\left(\mathrm{F}_{1,10}=6.19, p<0.05\right.$, power $\left.>0.60\right)$, and the mean CBV difference between techniques revealed by the Bland-Altman analysis $(1.7 \mathrm{ml} / 100 \mathrm{~g})$ was also significant $(p<0.001)$.

Figure 5 illustrates the predicted error in $\Delta \mu_{q_{3}}^{\max }$ caused by altering the FWHM of the IRF. As expected, ${ }^{23}$ the results show that the accuracy of $\Delta \mu_{a}^{\max }$ is very sensitive to instrument dispersion. An underestimation of $10 \%$ in the FWHM caused $\Delta \mu_{a}^{\max }$ to be overestimated by $11.6 \%$, while a $10 \%$ overestimation caused $\Delta \mu_{a}^{\max }$ to be underestimated by $20 \%$. The error analysis showed that the accuracy of $\Delta \mu_{a}^{\max }$ was insensitive to timing uncertainties and nonlinearities within the characterized range of the TR system. ${ }^{32}$ A 21 -ps error in the position of the IRF caused only a $2.2 \%$ error in $\Delta \mu_{a}^{\max }$. Also, the subtle change in the shape of the IRFs acquired at 


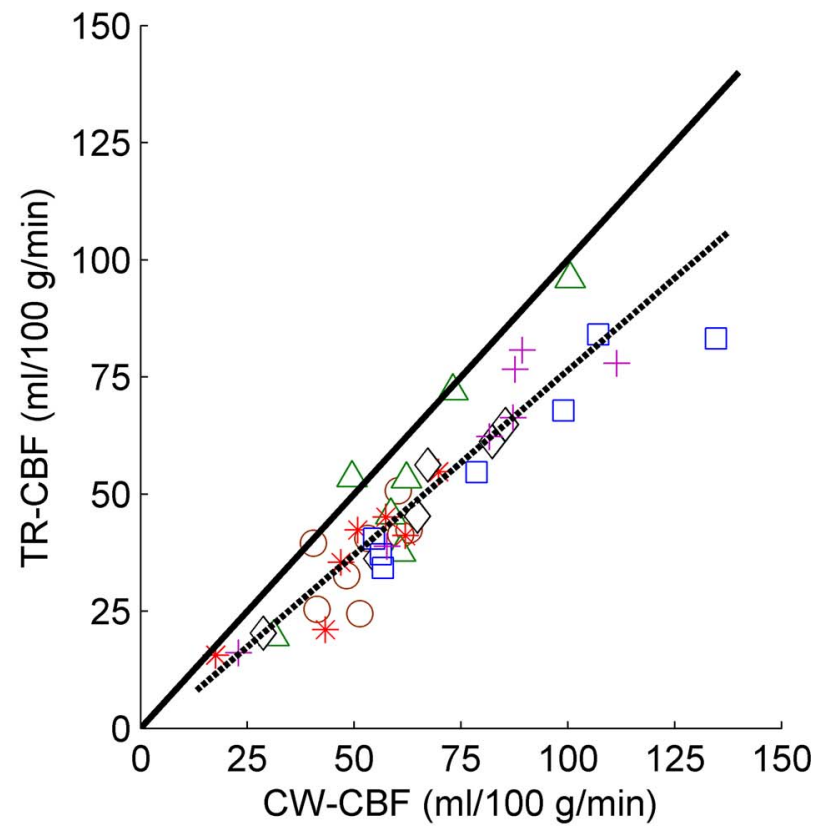

(a)

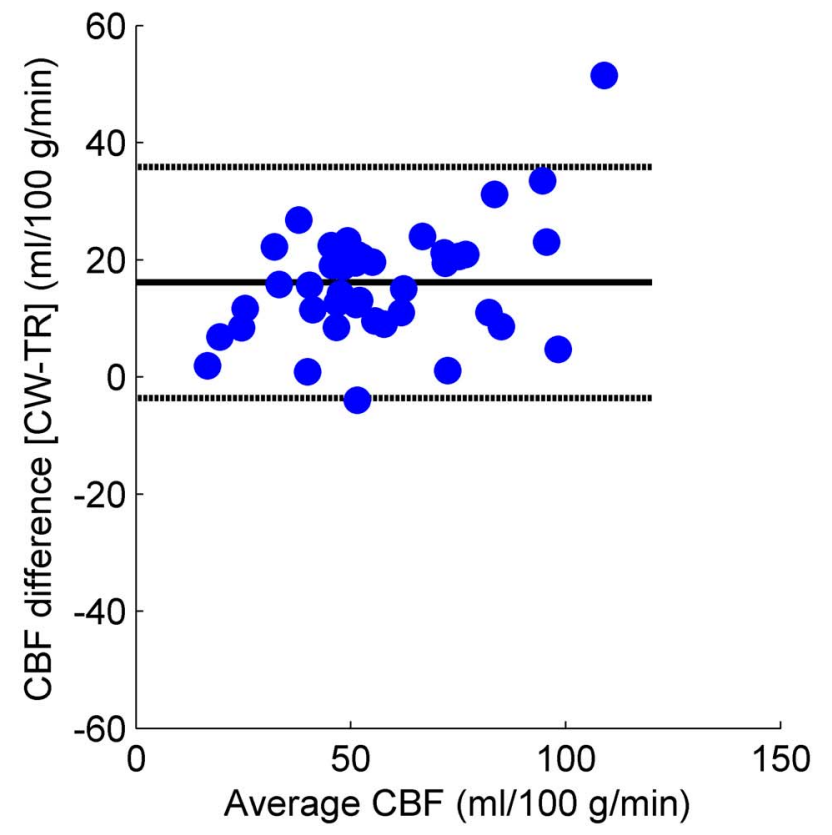

(b)

Fig. 3 (a) Correlation plot comparing CBF measurements acquired by continuous-wave and time-resolved techniques (labeled CW-CBF and TR-CBF, respectively). Each different colored symbol represents data acquired from one of the six piglets. The dashed line represents the average of all individual linear regressions (slope $=0.79$ and intercept $=-2.2 \mathrm{ml} / 100 \mathrm{~g} / \mathrm{min}$ ). The line of unity (solid line) is shown for comparison. (b) Bland-Altman plot showing the differences in CBF measurements from the two techniques. The mean difference was $16.1 \mathrm{ml} / 100 \mathrm{~g} / \mathrm{min}$ (solid line), and the limits of agreement, within which $95 \%$ of the differences reside, were -3.6 and $35.8 \mathrm{ml} / 100 \mathrm{~g} / \mathrm{min}$ (dashed lines).

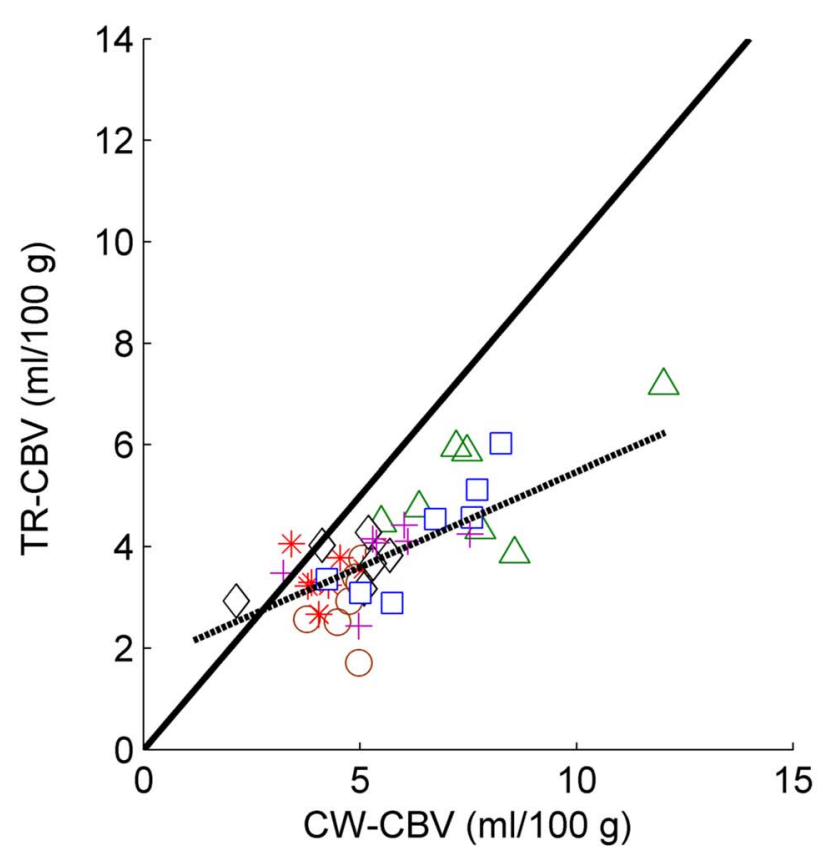

(a)

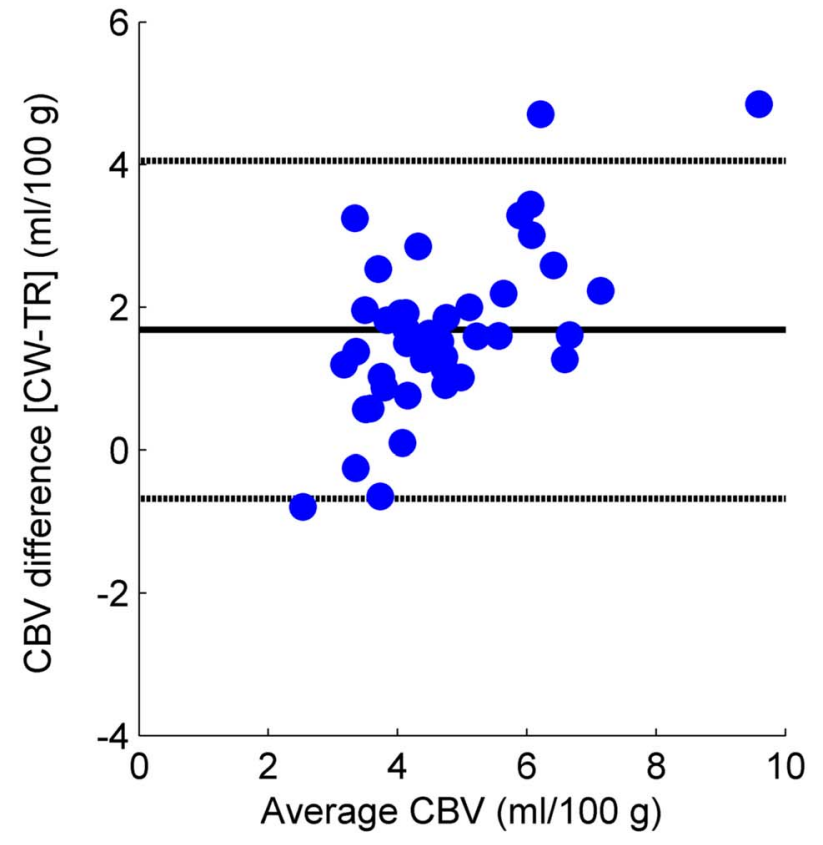

(b)

Fig. 4 (a) Correlation plot comparing CBV measurements acquired by continuous-wave and time-resolved techniques (labeled CW-CBV and TR-CBV, respectively). Each different colored symbol represents data acquired from one of the six piglets. The dashed line represents the average of all individual linear regressions (slope $=0.38$ and intercept $=1.72 \mathrm{ml} / 100 \mathrm{~g}$ ). The line of unity (solid line) is shown for comparison. (b) BlandAltman plot showing the differences in CBV measurements from the two techniques. The mean difference was $1.69 \mathrm{ml} / 100 \mathrm{~g}$ (solid line), and the limits of agreement were -0.68 and $4.05 \mathrm{ml} / 100 \mathrm{~g}$ (dashed lines). 
Diop et al.: Comparison of time-resolved and continuous-wave near-infrared techniques...

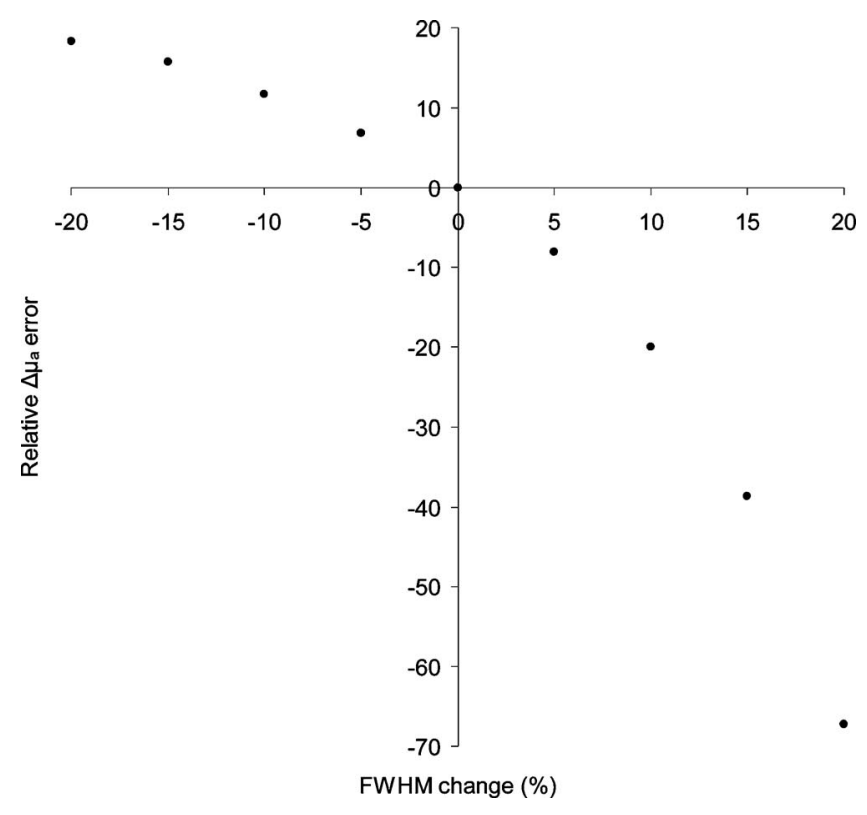

Fig. 5 Relative error in the measured maximum change in $\mu_{a}\left(\Delta \mu_{a}^{\max }\right)$ caused by altering the FWHM of the instrument response function (IRF). The FWHM of the true IRF was $0.490 \mathrm{~ns}$, and the input optical properties were $\mu_{s}^{\prime}=0.74 \mathrm{~cm}^{-1}$, baseline $\mu_{a}=0.150 \mathrm{~cm}^{-1}$, and $\Delta \mu_{\mathrm{a}}^{\max }=0.045 \mathrm{~cm}^{-1}$.

count rates 800 and $400 \mathrm{kHz}$ resulted in only a $-1.1 \%$ error in $\Delta \mu_{a}^{\max }$.

\section{Discussion}

Interest in biomedical applications of time-resolved NIR techniques has increased recently thanks in part to the development of relatively inexpensive high quality components, including pulsed diode lasers and compact dynode photomultiplier tubes. The main advantages of TR-NIR are the ability to quantify absorption and scattering properties, and improved depth discrimination using either time gating or higher temporal moments. ${ }^{15,18,37}$ Using the latter approach, the shape of brain ICG concentration curves was shown to reflect the extent of vessel stenosis in stroke patients, demonstrating the sensitivity of TR-NIR to cerebral perfusion. ${ }^{20}$ The focus of the current work was to build on these promising findings by developing a method for extracting quantitative CBF measurements from time-resolved ICG bolus-tracking data. Such a technique would be beneficial to the management of neurological emergencies, such as subarachnoid hemorrhage and traumatic brain injury, since ensuring adequate $\mathrm{CBF}$ during intensive care is critical for avoiding secondary brain injury. ${ }^{38}$

Our ICG bolus-tracking approach for measuring CBF is based on the same principles underlying contrast-enhanced MRI and CT techniques. ${ }^{39}$ The deconvolution method used to determine $\mathrm{CBF}$ from the dynamic arterial and tissue concentration curves has been validated in a CT perfusion study by comparison to blood flow measurements obtained by radiolabeled microspheres. ${ }^{11}$ We have also reported good agreement between $\mathrm{CBF}$ measurements acquired by $\mathrm{CT}$ perfusion and CW-NIRS in two previous studies involving newborn piglets. ${ }^{9,10}$ In the current study, a strong correlation $\left(\mathrm{R}^{2}\right.$ $=0.81)$ was observed between $\mathrm{CBF}$ measurements obtained by TR- and CW-NIRS methods over a flow range from 20 to $100 \mathrm{ml} / 100 \mathrm{~g} / \mathrm{min}$. The time-resolved approach also produced tissue ICG concentration curves with a high CNR (Fig. 2), although, as expected, the CNR was higher for CWNIRS. These results demonstrate that TR-NIR, combined with the bolus-tracking method, has the sensitivity to measure $\mathrm{CBF}$ over a clinically relevant range. However, $\mathrm{CBF}$ measurements from the two NIR techniques were not in perfect agreement, as indicated by the average correlation slope $(0.79 \pm 0.06)$ and the bias revealed by Bland-Altman analysis $(16.1 \mathrm{ml} / 100 \mathrm{~g} / \mathrm{min})$. A similar pattern was observed by comparing CBV measurements acquired by the two techniques. In this case, the correlation was only moderate and the average slope farther from unity. The poorer performance of the CBV comparison is partly explained by the greater variability in the measurements from both techniques and the smaller range of $\mathrm{CBV}$ values compared to the $\mathrm{CBF}$ range.

There are a number of potential sources of error with both techniques that could contribute to the discrepancy between the hemodynamic measurements. The continuous-wave method is clearly the simpler of the two techniques, but there are factors that can reduce its accuracy. The first consideration is the suitability of characterizing light attenuation by the modified Beer-Lambert law, since attenuation is a function of both absorption and scatter. However, it is reasonable to assume that the change in attenuation is linearly proportional to the change in absorption, provided the latter is relatively small: ${ }^{27}$ the amount of ICG injected was limited to $0.05 \mathrm{mg}$, resulting in a maximum change in the absorption coefficient of approximately $0.05 \mathrm{~cm}^{-1}$. A second concern is errors in the differential path length measurements obtained by seconddifferential analysis. Matcher, Cope, and Delpy ${ }^{27}$ demonstrated an excellent agreement between DP measurements obtained by this approach and values obtained with a Streak camera in a tissue-mimicking phantom. We determined the DP using the $840-\mathrm{nm}$ water feature, which is close to the maximum absorption wavelength of ICG in plasma. The differential path length factor calculated from the average DP and a source-detector distance of $2.7 \mathrm{~cm}$ was $3.69 \pm 0.22$, which is in good agreement with a previously reported value of 4.0, also obtained by CW-NIRS in newborn piglets. ${ }^{26}$ In both studies, the cerebral water concentration was assumed to be $85 \%$ due to the higher water concentration in the newborn brain compared to the adult brain. However, this value reflects gray matter and does not account for the lower water concentration in white matter, which is closer to $76 \%$ in piglets. ${ }^{40}$ The correct DP could be higher, depending on the proportional contributions of the two brain tissues in the collected reflectance. For example, adjusting DP for a 50/50 mixing of gray and white matter would reduce the $\mathrm{CW}-\mathrm{CBF}$ estimates by approximately $6 \%$. The CBF estimates would be further reduced using the larger time-resolved DP values. However, the corresponding water concentration would be $65 \%$, which is not physiologically possible in a healthy brain.

Given the complexity of the instrumentation, there are a number of factors that can degrade the accuracy of TR-NIR reflectance measurements. Temporal stability can be impaired by instrumental drift and jitter. ${ }^{23,41}$ From a series of instrument response functions acquired over a 3 -h period, we found that drift artifacts were negligible, provided the system was 
allowed a warm-up period of at least one hour. ${ }^{32}$ The effects of timing jitter were also quite small: the standard deviation of the temporal position of the IRF from a series of IRFs was $4.3 \mathrm{ps}^{32}$ An error in the position of the IRF as large as $21 \mathrm{ps}$ only caused a $2.2 \%$ error in the absorption change. Another common source of error is incorrectly characterizing the amount of dispersion caused by the system (i.e., the instrument response function). To measure the IRF, we followed previous recommendations and placed a thin piece of matte paper between the emission and detection probes to act as a diffuser. $^{24,25}$ The reasonable agreement between our measurements of the tissue-mimicking phantom's optical properties and the values provided by the manufacturer suggests that the IRF was properly characterized. The measured $\mu_{a}$ and $\mu_{s}^{\prime}$ values differed from the manufacturer's values (averaged over the two wavelengths) by 16 and $-20 \%$, respectively. It should be noted that errors in characterizing the shape of the IRF have significantly larger effects on $\mu_{s}^{\prime}$ than on $\mu_{a}$. For example, increasing the width of the IRF such that the timeresolved DP estimate equals the smaller CW-DP estimate resulted in only a $5 \%$ overestimation in $\mu_{a}$, but a $38 \%$ overestimation of $\mu_{s}^{\prime}$ (data not shown). Furthermore, the average values of $\mu_{a}, \mu_{s}^{\prime}$, and the differential path length factor of the piglet head $\left(0.176 \pm 0.001 \mathrm{~cm}^{-1}, 7.47 \pm 0.04 \mathrm{~cm}^{-1}\right.$, and $4.82 \pm 0.21$, respectively) were in good agreement with previous values. Using time-resolved spectroscopy and the same animal model, Kusaka et al. and Ijichi et al. reported $\mu_{a}=0.189 \pm 0.016 \mathrm{~cm}^{-1}, \mu_{s}^{\prime}=8.33 \pm 0.91 \mathrm{~cm}^{-1}$, and a differential path length factor $=5.3 \pm 0.32$ at $795 \mathrm{~nm}$ under normoxic conditions. ${ }^{30,42}$

Figure 5 demonstrates that underestimating $\Delta \mu_{a}^{\max }$, and conversely $\mathrm{CBF}$, would result from overestimating the FWHM of the IRF. However, this would also lead to an underestimation of the DP, since it is proportional to the difference in mean transit times between the experimental TPSF and the IRF. This scenario would seem unlikely, considering the time-resolved DP measurements are already significantly greater than the continuous-wave DP measurements. In fact, the more common occurrence is to underestimate instrument dispersion by not filling all modes of the detection fiber bundle. ${ }^{24}$ It is unlikely that light diffusion in the paper used to measure the IRF would have significant dispersion effects. To test this, we repeated the procedure with a thicker piece of paper, and no change in the shape of the IRF was observed (data not shown).

To correctly measure the tissue ICG concentration curve, the time-resolved system must be linear over the expected range of photon count rates. Of primary concern are distortions in the shape of the TPSF due to pile-up effects, since the magnitude of this artifact can vary with count rate. To minimize this potential source of error, the initial count rate (i.e., $800 \mathrm{kHz}$ ) was set to $1 \%$ of the pulse repetition rate, and the maximum reduction in the count rate was limited to approximately $400 \mathrm{kHz}$ by reducing the amount of ICG injected. Potential effects of distortions in the TPSF were assessed by measuring the IRF at the two extremes (400 and $800 \mathrm{kHz}$ ). The small error in $\Delta \mu_{a}^{\max }(-1.1 \%)$ resulting from differences between the two IRFs demonstrated that count-rate dependent distortions were negligible.

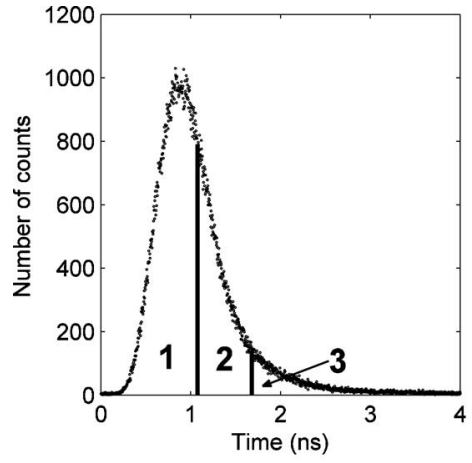

(a)

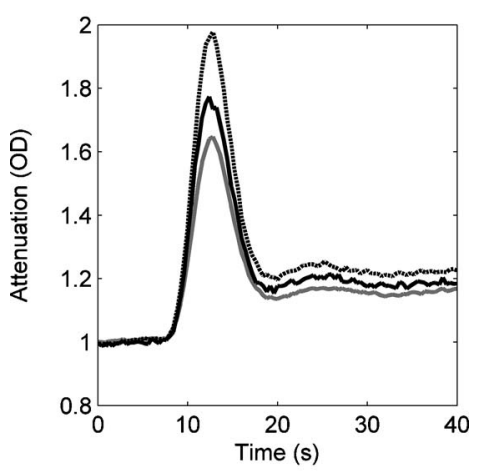

(b)

Fig. 6 (a) Typical TPSF acquired from a piglet head. Overlaid on the TPSF are the areas under the curve used to calculate attenuation changes for early (1), middle (2), and late (3) arriving photons, respectively. (b) Change in attenuation following an ICG injection for the three bins: early (gray curve), middle (dashed curve), and late arriving photons (solid line).

There are two plausible explanations for the differences found between the hemodynamic measurements from the two NIR techniques. First, although we were not able to clearly identify a single source of error strong enough to account for the discrepancy, it could be the result of a combination of small errors with both techniques. Examples would include uncertainties in the assumed water concentration required to measure DP by CW-NIRS, or variations between the measured and true IRF of the TR system. A second explanation is that the two techniques are interrogating slightly different brain regions. Intensity measurements by CW-NIRS are known to be more sensitive to tissues immediately below the surface, resulting in CBF measurements that, predominately reflect cortical gray matter. Since the cortex in the piglet brain is approximately $1 \mathrm{~cm}$ thick, the increased depth sensitivity of TR-NIR may have resulted in increased signal contributions from subcortical white matter, which has significantly lower blood flow. The DP measurements support this explanation, since the values obtained by the TR technique were greater than those obtained by CW spectroscopy-both in the current study and in previous studies involving piglets. ${ }^{26,30}$ Further evidence for this explanation is provided by the time-of-flight information. Figure 6 shows the change in attenuation following an ICG injection for three time windows: early, middle, and late. Intriguingly, the maximum attenuation change, which is proportional to $\mathrm{CBF}$, was found in the middle time 
interval, suggesting that photons in this time interval were probing cortical gray matter, while late-arriving photons were interrogating deeper white matter. Note that these attenuation curves were not converted into ICG concentration curves because of the substantial contribution of the IRF to the measured experimental TPSF (i.e., time-of-flight data). Since the IRF of our system was fairly wide, approximately $0.5 \mathrm{~ns}$, a deconvolution routine would be required to extract the true time-of-flight data. In future experiments, the difference in depth sensitivity between the two NIR techniques could be investigated using an imaging technique to acquire maps of CBF combined with modeling procedures to determine the light propagation paths.

In summary, this is the first study to attempt a quantitative measure of cerebral blood flow using time-correlated singlephoton technology. The technique produced ICG concentration curves with high $\mathrm{CNR}$, and could accurately track changes in $\mathrm{CBF}$ caused by either elevating arterial blood $\mathrm{CO}_{2}$ or impeding blood flow in the common carotids. A strong positive correlation between CBF measurements from the TRNIR technique and a previously validated CW-NIRS technique was found. Future work will include further validation in older animals with non-negligible extracerebral layers and adaptation to human studies. These applications will require incorporating a multilayered model ${ }^{43}$ or using time-of-flight data to improve depth sensitivity. Challenges facing these approaches include increased complexity of a layered model, signal contamination from ICG fluorescence at late arrival times, ${ }^{44}$ and errors in time-of-flight measurements due to the contribution of the IRF. The CBF measurements could also be combined with measurements of tissue oxygenation-derived from reflectance measurements at wavelengths sensitive to oxy- and deoxyhemoglobin concentrations - to determine the cerebral metabolic rate of oxygen. ${ }^{35,45}$

\section{Acknowledgments}

This study was supported through grants from the Canada Foundation for Innovation and the Ontario Federation of Cerebral Palsy. The authors would also like to thank Jennifer Hadway and Lise Desjardins for their help in conducting the animal experiments, and John Patrick and Lynn Keenliside for their technical support in building the time-resolved system.

\section{References}

1. A. Rocco, M. Pasquini, E. Cecconi, G. Sirimarco, M. C. Ricciardi, E. Vicenzini, M. Altieri, V. Di Piero, and G. L. Lenzi, "Monitoring after the acute stage of stroke: a prospective study," Stroke 38(4), 1225 1228 (2007).

2. A. Helmy, M. Vizcaychipi, and A. K. Gupta, "Traumatic brain injury: intensive care management," Br. J. Anaesth. 99(1), 32-42 (2007).

3. C. N. Gallagher, P. J. Hutchinson, and J. D. Pickard, "Neuroimaging in trauma," Curr. Opin. Neurol. 20(4), 403-409 (2007).

4. W. L. Wright, "Multimodal monitoring in the ICU: when could it be useful?," J. Neurol. Sci. 261, 10-15 (2007).

5. P. Bolognese, J. I. Miller, I. M. Heger, and T. H. Milhorat, "LaserDoppler flowmetry in neurosurgery," J. Neurosurg. Anesthesiol. 5(3), 151-158 (1993).

6. M. A. De Georgia and A. Deogaonkar, "Multimodal monitoring in the neurological intensive care unit," Neurologist 11(1), 45-54 (2005).

7. F. F. Jobsis, "Noninvasive, infrared monitoring of cerebral and myocardial oxygen sufficiency and circulatory parameters," Science 198(4323), 1264-1267 (1977).
8. P. G. Al-Rawi, "Near infrared spectroscopy in brain injury: today's perspective," Acta Neurochir. Suppl. 95, 453-457 (2005).

9. D. W. Brown, P. A. Picot, J. G. Naeini, R. Springett, D. T. Delpy, and T. Y. Lee, "Quantitative near infrared spectroscopy measurement of cerebral hemodynamics in newborn piglets," Pediatr. Res. 51(5), 564 (2002).

10. M. Diop, J. T. Elliott, K. M. Tichauer, T. Y. Lee, and K. St. Lawrence, "A broadband continuous-wave multichannel nearinfrared system for measuring regional cerebral blood flow and oxygen consumption in newborn piglets," Rev. Sci. Instrum. 80(5), 054302 (2009).

11. A. Cenic, D. G. Nabavi, R. A. Craen, A. W. Gelb, and T. Y. Lee, "Dynamic CT measurement of cerebral blood flow: a validation study," AJNR Am. J. Neuroradiol. 20(1), 63 (1999).

12. K. L. Zierler, "Equations for measuring blood flow by external monitoring of radioisotopes," Circ. Res. 16, 309 (1965).

13. F. Gora, S. Shinde, C. E. Elwell, J. C. Goldstone, M. Cope, D. T. Delpy, and M. Smith, "Noninvasive measurement of cerebral blood flow in adults using near-infrared spectroscopy and indocyanine green: a pilot study," J. Neurosurg. Anesthesiol. 14(3), 218-222 (2002).

14. R. Mudra, C. Muroi, P. Niederer, and E. Keller, "Near-infrared spectroscopy extended with indocyanine green dye dilution for cerebral blood flow measurement: median values in healthy volunteers,' Opto-Electron. Rev. 16(3), 297-308 (2008).

15. J. Selb, J. J. Stott, M. A. Franceschini, A. G. Sorensen, and D. A. Boas, "Improved sensitivity to cerebral hemodynamics during brain activation with a time-gated optical system: analytical model and experimental validation," J. Biomed. Opt. 10(1), 11013 (2005).

16. A. Liebert, H. Wabnitz, J. Steinbrink, H. Obrig, M. Moller, R. Macdonald, A. Villringer, and H. Rinneberg, "Time-resolved multidistance near-infrared spectroscopy of the adult head: intracerebral and extracerebral absorption changes from moments of distribution of times of flight of photons," Appl. Opt. 43(15), 3037-3047 (2004).

17. M. Kohl-Bareis, H. Obrig, J. Steinbrink, J. Malak, K. Uludag, and A. Villringer, "Noninvasive monitoring of cerebral blood flow by a dye bolus method: separation of brain from skin and skull signals," $J$. Biomed. Opt. 7(3), 464-470 (2002).

18. B. Montcel, R. Chabrier, and P. Poulet, "Detection of cortical activation with time-resolved diffuse optical methods," Appl. Opt. 44(10), 1942-1947 (2005).

19. J. Steinbrink, T. Fischer, H. Kuppe, R. Hetzer, K. Uludag, H. Obrig, and W. M. Kuebler, "Relevance of depth resolution for cerebral blood flow monitoring by near-infrared spectroscopic bolus tracking during cardiopulmonary bypass," J. Thorac. Cardiovasc. Surg. 132(5), 1172-1178 (2006).

20. A. Liebert, H. Wabnitz, J. Steinbrink, M. Moller, R. Macdonald, H. Rinneberg, A. Villringer, and H. Obrig, "Bed-side assessment of cerebral perfusion in stroke patients based on optical monitoring of a dye bolus by time-resolved diffuse reflectance," Neuroimage 24(2), 426-435 (2005).

21. A. Kienle and M. S. Patterson, "Improved solutions of the steadystate and the time-resolved diffusion equations for reflectance from a semi-infinite turbid medium," J. Opt. Soc. Am. A Opt. Image Sci. Vis 14(1), 246-254 (1997).

22. W. Becker, Advanced Time-Correlated Single Photon Counting Techniques, Springer, New York (2005).

23. V. Ntziachristos and B. Chance, "Accuracy limits in the determination of absolute optical properties using time-resolved NIR spectroscopy," Med. Phys. 28(6), 1115-1124 (2001).

24. A. Liebert, H. Wabnitz, D. Grosenick, and R. Macdonald, "Fiber dispersion in time domain measurements compromising the accuracy of determination of optical properties of strongly scattering media," J. Biomed. Opt. 8(3), 512-516 (2003).

25. E. Alerstam, S. Andersson-Engels, and T. Svensson, "White Monte Carlo for time-resolved photon migration," J. Biomed. Opt. 13(4), 041304 (2008)

26. R. Springett, Y. Sakata, and D. T. Delpy, "Precise measurement of cerebral blood flow in newborn piglets from the bolus passage of indocyanine green," Phys. Med. Biol. 46(8), 2209-2225 (2001).

27. S. J. Matcher, M. Cope, and D. T. Delpy, "Use of the water absorption spectrum to quantify tissue chromophore concentration changes in near-infrared spectroscopy," Phys. Med. Biol. 39(1), 177 (1994). 
28. E. Alerstam, S. Andersson-Engels, and T. Svensson, "Improved accuracy in time-resolved diffuse reflectance spectroscopy," Opt. Express 16(14), 10440-10454 (2008).

29. M. L. Landsman, G. Kwant, G. A. Mook, and W. G. Zijlstra, "Lightabsorbing properties, stability, and spectral stabilization of indocyanine green," J. Appl. Physiol. 40(4), 575-583 (1976).

30. T. Kusaka, Y. Hisamatsu, K. Kawada, K. Okubo, H. Okubo, M. Namba, T. Imai, K. Isobe, and S. Itoh, "Measurement of cerebral optical pathlength as a function of oxygenation using near-infrared time-resolved spectroscopy in a piglet model of hypoxia," Opt. Rev. 10(5), 466-469 (2003).

31. D. T. Delpy, M. Cope, P. van der Zee, S. Arridge, S. Wray, and J. Wyatt, "Estimation of optical pathlength through tissue from direct time of flight measurement," Phys. Med. Biol. 33(12), 1433-1442 (1988).

32. M. Diop, J. T. Elliott, K. M. Tichauer, M. Migueis, T. Y. Lee, and K. St. Lawrence, "Bedside monitoring of absolute cerebral blood flow by time-resolved NIRS," Proc. SPIE 7555, 1-9 (2010).

33. K. M. Tichauer, D. W. Brown, J. Hadway, T. Y. Lee, and K. St. Lawrence, "Near-infrared spectroscopy measurements of cerebral blood flow and oxygen consumption following hypoxia-ischemia in newborn piglets," J. Appl. Physiol. 100(3), 850-857 (2006).

34. S. L. Zeger, K. Y. Liang, and P. S. Albert, "Models for longitudinal data: a generalized estimating equation approach," Biometrics 44(4), 1049-1060 (1988)

35. K. M. Tichauer, J. A. Hadway, T. Y. Lee, and K. St. Lawrence, "Measurement of cerebral oxidative metabolism with near-infrared spectroscopy: a validation study," J. Cereb. Blood Flow Metab. 26(5), 722-730 (2006)

36. J. M. Bland and D. G. Altman, "Statistical methods for assessing agreement between two methods of clinical measurement," Lancet 1(8476), 307-310 (1986).

37. A. Liebert, H. Wabnitz, D. Grosenick, M. Moller, R. Macdonald, and H. Rinneberg, "Evaluation of optical properties of highly scattering media by moments of distributions of times of flight of photons," Appl. Opt. 42(28), 5785-5792 (2003).

38. J. J. Kraus, M. D. Metzler, and W. M. Coplin, "Critical care issues in stroke and subarachnoid hemorrhage," Neurol. Res. 24(Suppl 1), S47-57 (2002).

39. M. Wintermark, M. Sesay, E. Barbier, K. Borbely, W. P. Dillon, J. D. Eastwood, T. C. Glenn, C. B. Grandin, S. Pedraza, J. F. Soustiel, T. Nariai, G. Zaharchuk, J. M. Caille, V. Dousset, and H. Yonas, "Comparative overview of brain perfusion imaging techniques," Stroke 36(9), e83-99 (2005).

40. V. C. Rose, D. H. Shaffner, C. A. Gleason, R. C. Koehler, and R. J. Traystman, "Somatosensory evoked potential and brain water content in post-asphyxic immature piglets," Pediatr. Res. 37(5), 661-666 (1995).

41. F. E. Schmidt, M. E. Fry, E. M. Hillman, J. C. Hebden, and D. T. Delpy, "A 32-channel time-resolved instrument for medical optical tomography," Rev. Sci. Instrum. 71(1), 256-265 (2000).

42. S. Ijichi, T. Kusaka, K. Isobe, F. Islam, K. Okubo, H. Okada, M. Namba, K. Kawada, T. Imai, and S. Itoh, "Quantification of cerebral hemoglobin as a function of oxygenation using near-infrared timeresolved spectroscopy in a piglet model of hypoxia," J. Biomed. Opt. 10(2), 024026 (2005).

43. A. Kienle, M. S. Patterson, N. Dognitz, R. Bays, G. Wagnieres, and H. van den Bergh, "Noninvasive determination of the optical properties of two-layered turbid media," Appl. Opt. 37(4), 779-791 (1998).

44. O. Steinkellner, H. Wabnitz, A. Jelzow, C. Gruber, J. Steinbrink, H. Obrig, and R. Macdonald, "Instrumentation and methodology for bedside monitoring of cerebral perfusion by optical bolus tracking," Biomed. Opt. OSA Tech. Digest (CD), paper BSuD94 (2010).

45. D. W. Brown, J. Hadway, and T. Y. Lee, "Near-infrared spectroscopy measurement of oxygen extraction fraction and cerebral metabolic rate of oxygen in newborn piglets," Pediatr. Res. 54(6), 861-867 (2003). 